\title{
Responsabilidad penal para los y las Adolescentes: Análisis de la ideología del control penal, garantía de derechos e idealismo penal
}

\author{
Criminal responsibility for adolescents: Analysis of criminal control \\ ideology, guarantee of rights and penal idealism
}

Autores: Estanislao Escalante, Michael Stiven Reyes Barreto DOI: https://doi.org/10.25058/1794600X.1797 


\title{
RESPONSABILIDAD PENAL PARA LOS Y LAS ADOLESCENTES: ANÁLISIS DE LA IDEOLOGÍA DEL CONTROL PENAL, GARANTÍA DE DERECHOS E IDEALISMO PENAL*
}

\author{
Criminal responsibility for adolescents: Analysis of criminal \\ control ideology, guarantee of rights and penal idealism \\ Responsabilidade criminal para adolescentes: Análise da \\ ideologia do controle criminal, garantia de direitos e idealismo
}

criminal

\author{
Estanislao Escalante ${ }^{a}$ \\ ceescalanteb@unal.edu.co \\ Michael Stiven Reyes Barreto ${ }^{b}$ \\ msreyesb@unal.edu.co
}

Fecha de recepción: 11 de mayo de 2020 Fecha de revisión: 18 de mayo de 2020

Fecha de aceptación: 27 de junio de 2020

\section{RESUMEN}

DOI: http://doi.org/10.25058/1794600X.1797

Para citar esta colaboración especial:

Escalante, E. y Reyes, M. S. (2020). Responsabilidad penal para los y las Adolescentes: Análisis de la ideología del control penal, garantía de derechos e

idealismo penal. Revista Misión Jurídica, 13, (19). 252-269.

En el presente artículo se hace un balance de la implementación, comprensión y aplicación del Sistema de Responsabilidad Penal para Adolescentes en Colombia y se dilucidan los discursos punitivos, los mediáticos y los ideológicos en contraste con la evidencia empírica que muestra la brecha entre derecho y realidad, argumentando que las finalidades establecidas en la normatividad, que promulgó este sistema, no han tenido otro efecto que el de ocultar las falencias estructurales de nuestra sociedad.

\section{PALABRAS CLAVE}

Delincuencia juvenil, Sistema Penal, Control social, Política criminal, Criminología mediática, Proceso penal, Responsabilidad Penal Juvenil.

\footnotetext{
* Artículo de investigación.

a. Abogado, magister en Derecho, en Estudios de corrupción y estado de derecho y en pedagogía en la línea de docencia Universitaria. Profesor e investigador asociado de la Facultad de Derecho, Ciencias Políticas y Sociales de la Universidad Nacional de Colombia. Director de la Escuela de Investigación en Criminologías Críticas, Justicia Penal y Política Criminal "Luis Carlos Pérez" - Polcrymed- y del observatorio "Medios de comunicación, política criminal y justicia penal" de la Universidad Nacional de Colombia.
}

b. Abogado de la Universidad Nacional de Colombia, auxiliar docente en la cátedra de derecho penal general. Investigador de la Escuela de Investigación en Criminologías Críticas, Justicia Penal y Política Criminal "Luis Carlos Pérez" - Polcrymed-. 


\section{ABSTRACT}

This article considers the implementation, comprehension and application of the Criminal Responsibility System for Adolescents in Colombia and elucidates the punitive, media, and ideological discourses in contrast with empirical evidence that shows the gap between law and reality, arguing that the purposes established in regulations that this system enacted had no other effect than to hide the structural flaws in our society.

\section{KEYWORDS}

Juvenile crime; penal system; social control; criminal policy; media criminology; criminal proceeding; juvenile criminal responsibility.

\section{RESUMO}

Neste artigo, faz-se um balanço da implementação, compreensão e aplicação do Sistema de Responsabilidade Penal para Adolescentes na Colômbia e os discursos punitivos, midiáticos e ideológicos são elucidados em contraposição à evidência empírica que mostra a lacuna entre o direito e a realidade, argumentando que as finalidades estabelecidas no regulamento, promulgado por este sistema, não surtiram outro efeito que ocultar as falhas estruturais de nossa sociedade.

\section{PALAVRAS-CHAVE}

Delinquência juvenil; sistema penal, controle social, política criminal, criminologia da mídia, processo penal, responsabilidade penal juvenil.

\section{INTRODUCCIÓN}

La Ley 1098 de 2006 estableció en Colombia el Sistema de Responsabilidad penal para Adolescentes (SRPA) y a pesar de su efectiva implementación, el balance de su comprensión y aplicación no es claro: un sector de la sociedad considera que promueve la impunidad al dejar en libertad a adolescentes con un alta incidencia en la comisión de conductas con características delictivas, asimismo que la suavidad de las sanciones es aprovechada por el crimen organizado, nutriéndose cada vez más de la infancia para ocupar sus filas ${ }^{1}$; mientras que el otro sector, critica la brecha entre derecho y realidad, pues aseguran que las finalidades establecidas en la normatividad que promulgó este sistema, no han tenido otro efecto que el de ocultar las falencias estructurales de nuestra sociedad y en cambio sí han profundizado la criminalización del más vulnerable.

Cada una de estas posturas está respaldada por un postulado legitimador del sistema penal. La primera, responde al problema desde la perspectiva punitiva y retribución, renunciando a la rehabilitación del sujeto infractor, pues mira todas las medidas con agnosticismo. La segunda, comparte esta mirada agnóstica, aunque no descarta la resocialización, sino que crítica su imposibilidad material. Sin embargo, ninguna de estas posturas es congruente con la Constitución ni con los instrumentos internacionales sobre los derechos de la infancia, pues se limitan a cuestionar las falencias sin proponer soluciones alternativas y constitucionalmente orientadas.

No obstante, con esto no se quiere decir que no exista una disociación entre derecho y realidad, pues la sanción pedagógica es una clara muestra de ello. Los centros de reclusión ${ }^{2}$, para adolescentes no son compatibles con el anunciado fin pedagógico de la sanción: en un mismo espacio se imparten clases para personas de diferentes edades y grados escolares, develando claramente la carencia de reflexión del acto educativo por parte del pedagogo, que más que educador se

1. Sobre la forma como se han mediatizado las conductas de los adolescentes, los procesos de reacción social, mediática y la construcción del adolescente como un peligro para la sociedad se puede leer en nuestro artículo "Justicia Penal Juvenil: Negación de un Mandamiento Constitucional desde el Discurso Mediático y Político del Delito y la Defensa Social", (Escalante, 2018), en el mismo libro de investigación (Escalante, Política Crimina Mediática, Populismo Penal, Criminología Crítica de los Medios y de la Justicia Penal, 2018) se pueden leer los artículos "Narrativas, punitivismo y control social de adolescentes" (Romero, 2018), "Criminalización del adolescente en el modelo neoliberal: una mirada latinoamericana" (Díaz Mejía, 2018) y finalmente, el artículo "Infancia y mediatización entre la prescindibilidad, el peligrosísmo y el uso mercantil de las infancias y adolescencias" (Martínez A, 2018)

2. La Ley 1098 de 2006 los denomina como Establecimiento de Atención Especializada en el que cumplen la sanción privativa de la libertad los adolescentes que han sido sancionados en el SRPA. Sin embargo, desde una perspectiva crítica y atendiendo a la realidad de la privación de la libertad en Colombia y encontrada en diversos informes, tanto de le Defensoría del Pueblo como de la misma Procuraduría General de la Nación, se trata de verdaderos centros de reclusión para Niños, Niñas y Adolescentes. De manera que, para superar este tipo de idealismos y normalización del discurso se entiende que los CAE son Centros de Reclusión. 
convierte en un vigilante que imparte orden y disciplina (Defensoría del pueblo, 2015) ${ }^{3}$. Como se observa en el informe de la Defensoría del Pueblo (2015) y en los trabajos de campo realizados a los diversos Centros de Atención Especializada, estos centros de encerramiento, además de ser centros de disciplina, control, castigo y represión, son verdaderas cárceles para niños y niñas, que en lugar de realizar un nuevo proyecto de vida, les cosifica y cada centro de internamiento se convierte en una máquina perfecta para la reproducción de la pobreza, para la exclusión social y para la iniciación en su carrera criminal.

Estas condiciones no permiten que el adolescente reflexione sobre sus actos, que adopte los valores de un Estado Social de Derecho o asuma un comportamiento de respeto a los demás y se motive reflexivamente conforme a las expectativas sociales de comportamiento, sino que, como en el caso de las cárceles para adultos, inicie o continúe una carrera criminal, sin posibilidades para pensar y forjar un proyecto de vida distinto (Defensoría del pueblo, 2015). No se puede llegar a otra conclusión cuando el mismo informe de la Defensoría del Pueblo denuncia el uso de antiguas cárceles para privar de la libertad a los adolescentes, castigos físicos para el control disciplinario, el uso de celdas de aislamiento como castigo o infraestructura carcelaria como parte de lo que la ley, las autoridades y los expertos llaman en una especie de idealización ciega: Centros de Atención Especializados.

Ahora bien, los medios de comunicación y los políticos en ejercicio demandan mayores "penas" para los niños, niñas y adolescentes, y orientan su discurso desde una perspectiva penal, con énfasis en el control punitivo de la pena (Escalante, 2018), de manera que abordan su discurso mediático desde la perspectiva de las funciones de la pena del código penal, sin reparar en las diferencias que convencional y ontológicamente existen entre los adultos y los niños ${ }^{4}$.

3. Parte de las conclusiones que acá se exponen, hacen parte del trabajo de investigación que realiza la Escuela de Investigación en Criminologías críticas, Justicia Penal y Política criminal, Polcrymed de la Universidad Nacional de Colombia y también de la participación activa de uno de los autores en el primer informe de la Defensoría del Pueblo sobre el SRPA ante el Congreso de la República y la visita a los centros de atención especializada y de internamiento preventivo del SRPA en el país.

4. En este texto se entiende el concepto de niño como toda persona menor de edad (18 años) sin distinguir entre las diversas etapas de su desarrollo. De acuerdo con la Constitución y las sentencias
Este texto propone extrapolar las conclusiones sobre los estudios de la pena en el sistema penal para adultos, los cuales han planteado históricamente, por parte de sus más informados críticos, que la pena no cumple los fines que pretende cumplir, sino que cumple funciones latentes (Zaffaroni, 2016) o ideológicas (Baratta, 2002), por lo cual se toma distancia del ideal resocializador, sin que esto signifique una adopción de políticas neo punitivas, que sólo ahondan en el problema a través de medidas que progresivamente desconocen los derechos humanos. Tampoco buscamos confundir las funciones de la pena de los adultos con las finalidades de la sanción pedagógica, educativa y restaurativa del sistema de adolescentes, sino que, al contrario, buscamos demostrar cómo la fundamentación de un derecho penal para adolescentes basado en las funciones de la pena es idealismo puro y somete toda la práctica pedagógica del sistema de adolescentes a la ideología de la defensa social (Baratta, 2002).

Por tanto, se cambiará el énfasis de la sanción para pasar a estudiar el proceso como escenario pedagógico, en el cual todos los actores del proceso (incluyendo al adolescente) participen en la interiorización y comprensión de los valores democráticos, evitando siempre, y en la mayor medida posible, la reclusión de los adolescentes, ya que como se ha expuesto no hace más que ocultar, por medio de nombres eufemísticos, un castigo que puede ser peor que el de los adultos, debido a la manta aún presente del paternalismo y a la huella especulativa del idealismo penal.

Con este propósito, en primer lugar, se hará un recuento histórico sobre los orígenes del derecho penal, caracterizándolo como un derecho penal inhumano ${ }^{5}$. En segundo lugar, se abordará la infancia como un concepto naturalizado sobre el cual se han construido dos paradigmas principales

de constitucionalidad, es niño, como sujeto de derechos, toda persona menor de edad de acuerdo a la normatividad vigente. La distinción de menor con el de niñez, como se verá, está revalorada en la teoría al superarse el paradigma de la situación irregular, pero muy arraigado en los servidores y actores del sistema en la forma de concebir al menor de edad como objeto de protección, objeto del derecho o de las políticas públicas. (García Méndez, 2017)

5. La importancia de este análisis radica en que algunos de los servidores del SRPA e incluso los defensores públicos del sistema de defensoría pública, han manifestado que este sistema es un sistema penal de adultos para "chiquitos" y así actúan en él y ejecutan el mismo. Entre otros, ver en (Defensoría del pueblo, 2015), (Escalante, 2018; Martínez A, 2018). 
de responsabilidad penal ${ }^{6}$. En tercer lugar, se expondrá el deber ser colombiano del Sistema de Responsabilidad Penal para Adolescentes. Finalmente se llenará de contenido un precepto parcialmente olvidado: el proceso penal como escenario pedagógico.

\section{DERECHO PENAL INHUMANO}

A lo largo de la historia del pensamiento penal, algunos autores han asegurado que el derecho penal es aséptico y ahistórico, que la verdadera ciencia penal es la que prescinde de elementos sociológicos que suelen estropear la tan anhelada coherencia normativa (Rocco, 1986) ${ }^{7}$. Sin embargo, esta visión no tiene en cuenta que la dogmática penal es el resultado de un ejercicio político, influido por un contexto histórico determinado, el cual responde a una realidad específica (Zaffaroni, 2017, p. 27-28).

En el contexto latinoamericano, el derecho penal ha sido el producto de importaciones acríticas que no tienen presente el carácter político de estas elaboraciones dogmáticas. Por ejemplo, la importación del neokantismo, que sirvió como recurso al vacío que dejó la perdida de legitimidad de la criminología positivista en la posguerra, se debió a cierto tipo de colonialismo jurídico que se remontaba al siglo anterior (Zaffaroni, Doctrina Penal Nazi, la Dogmática Penal Alemana entre 1933 y 1945, 2017, págs. 161-163).

El siglo XIX se caracterizó por la consolidación de nuevos actores y saberes, la sociedad europea era seducida por las ciencias exactas. El método científico de las ciencias naturales se impuso

6. García Méndez (2017) indica que la doctrina de la situación irregular y el paradigma de la protección integral son los paradigmas principales, aunque precisa que, como resultado de la indebida aplicación del último, surge el paradigma de la ambigüedad. No compartimos que se trate propiamente de un paradigma científico en el marco de la comunidad científica o en el estado del arte y mucho menos en los términos de Thomas Kuhn como revolución científica (Kuhn, 2013), pero si de una creciente tendencia crítica de los autores más informados, postura según la cual, el sistema no cumple ni los fines, ni las funciones atribuidas y en cambio sí está generando un creciente direccionamiento hacía la criminalización temprana de los adolescentes en América Latina en general, en Colombia en particular.

7. Al respecto, un análisis del problema del método penal, de la dogmática y de las teorías europeas en Massimo DONINI. (2011). Para ver el tránsito del actual sistema penal acusatorio en Colombia desde la preeminencia del derecho canónigo, pasando por el derecho germano y anglosajón, García y Serrano (2008) en todos los campos de conocimiento, sin diferenciar los objetos de estudio (Vega, 2011, p. 58). Esta aproximación epistemológica influyó directamente en el estudio del derecho penal, que hasta la fecha se había fundamentado en la filosofía liberal del iluminismo.

La implementación de este método derivó en elaboraciones teóricas de carácter descriptivo, como en el caso de Cesare Lombroso (Lombroso, 1909), quien a partir de la observación detenida de cierta población carcelaria llegó a la conclusión de que es equivocado asegurar que algunos sujetos pueden determinarse libremente frente a diferentes situaciones, lo cual consideró una ilusión racionalista heredada de un deductivismo ciego (Baratta, 2002, p. 31-32). De esta manera "En el positivismo criminológico, el delito tiene sus causas en el delincuente. La maldad tiene nombre propio y las causas serán en el mayor de los casos biológicas" (Gómez Jaramillo, 2008: 78).

No obstante, esto significaba que la corporación médica era quien determinaba la labor de los juristas, la criminología positivista ordenaba al derecho penal. Según Zaffaroni (2016), esto incomodó enormemente a los juristas, quienes limitaron epistemológicamente a la criminología, apartándola como una mera ciencia auxiliar. De esta manera, la ciencia jurídico penal recobró el primer plano, lo que significó una vuelta al idealismo (p.28).

Sin embargo, existe cierta continuidad de la Escuela de Badén (neokantismo) con las elaboraciones dogmáticas anteriores. No se trata de una negación total, sino de la introducción de valores a un esquema ya consolidado: el esquema liszt-Beling. Asimismo, conviene subrayar los esfuerzos de Von Liszt por implementar las instrucciones de la criminología positivista, pues introdujo conceptos sociológicos y creó, a su vez, la Escuela de Marburgo. Esta, a partir del positivismo crítico, expuso la necesidad de establecer fines a la pena, dejando así de ser exclusivamente concebida desde la retribución enseñada por los filósofos Kant y Hegel (Rivera, 2005: 82-83). De ahí que, a través de la introducción de elementos "ónticos", se le asignara funciones irrealizables a la pena, configurándose de esta manera un idealismo accidental

Por otra parte, el neokantismo responde a la teoría de conocimiento kantiana que corresponde 
a la crítica de la razón práctica. De donde resulta que las ciencias del espíritu (en las cuales el derecho está inmerso) parten de una realidad desorganizada. Según los neokantianos, se hacen indispensables los valores para organizar el caos que representa el objeto de las ciencias culturales (Zaffaroni, 2016: 27).

Ahora bien, es sabido que la interpretación de la Escuela de Badén no es del todo fiel al pensamiento kantiano, pues mientras para Kant la cosa en sí gozaba de existencia -aunque sólo pudiera ser alcanzada a través de la mediación de su entendimiento-, para los neokantianos no existía, por lo que los valores crean y ordenan los entes que valoran, constituyéndose así un idealismo valorativo desbordado (Vega, 2011: 60).

Simultáneamente a estas elaboraciones dogmáticas, la segunda guerra mundial llegó a su fin con el proceso reflexivo que conllevó. Las barbaridades legitimadas por una maquinaria de pensamiento racista fueron expuestas con perplejidad al descubrir su fundamentación legal. En definitiva, se acudió a todo tipo de iusnaturalismos con el propósito de limitar la omnipotencia del legislador. No obstante, esta forma de limitar el poder punitivo no satisfizo a Hans Welzel, quien propuso una forma de limitación más sencilla: las estructuras lógicoobjetivas (Zaffaroni, 2016).

Para Welzel, las herramientas conceptuales ofrecidas por el iusnaturalismo y el positivismo resultaban insuficientes. El positivismo de Kelsen centraba la validez de las normas únicamente en la forma como se hubiesen expedido -lo cual fácilmente constituye una brecha para legitimar cualquier visión inhumana del derecho penal-. Mientras que el iusnaturalismo argumentaba que la validez de las normas no dependía de la forma, sino de la coherencia de contenido con valores extrajurídicos. Sin embargo, el autor alemán recalca que la fundamentación teórica del iusnaturalismo no representa sino una justificación de un programa de acción ideal que se espera que los individuos acepten, por lo cual se estaría vendiendo una visión ideológica del derecho (Agudelo Betancur, 1998: 113-116). De modo que era necesario mirar otra forma de limitación al poder punitivo que se apartara de estas teorías del derecho (Welzel, 1951: 18).
Con esto en mente, Welzel acudió a la filosofía realista aristotélica, la cual intentaba acercar las ideas de Platón a la realidad sensible. Para Aristóteles, las cosas que se observan están compuestas de tres elementos: la substancia, la esencia y lo accidental. El primer elemento, se refiere a lo que yace debajo de la cosa, de lo cual se predica la unidad de todas las características que la componen, en otras palabras, es el núcleo. El segundo elemento, describe las características sin las cuales la cosa dejaría de serlo. Por último, lo accidental es lo que se puede prescindir sin que se afecte la identidad del ente (Vega, 2011: 64). Un ejemplo de esto lo podemos encontrar en la clasificación de los elementos del contrato realizada por Andrés Bello.

Asimismo, Welzel hizo uso no reconocido de la obra de Hartmann, precisando la consideración del filósofo griego que consideraba que las cosas tienen una finalidad inherente. En contraste, aseguró que las cosas no tienen una finalidad inmanente, característica que reservó a la conciencia (Agudelo Betancur, 1998).

En resumen, se puede afirmar que la obra del comentado tratadista alemán revolucionó la teoría del delito, que hasta el momento se hallaba inmersa en un idealismo valorativo que negaba cualquier inclusión de datos ónticos. Además, por medio de la inclusión de las estructuras lógicoobjetivas puso de presente que existe una realidad que el derecho no puede pasar por alto si aspira a ser eficaz, lo cual implicó un límite preciso a la creencia de que lo valorado creaba el objeto de la valoración.

A pesar de los esfuerzos realizados en la teoría del delito, Welzel no se apartó de la doctrina común respecto a la teoría de la pena, la cual se remonta a 1830, cuando Anton Bauer clasificó las penas en absolutas y relativas, las primeras correspondían a una deducción y las últimas tenían como propósito disuadir, intimidar y neutralizar (Zaffaroni, 2015). No obstante, esta elaboración no respondía a una investigación sociológica, sino a un ejercicio de mera deducción. En palabras de Zaffaroni (2015): “(...) el penalista suele decidir primero para qué y cómo debe ser la pena, para luego y en base a esa decisión construir toda su teoría penal según ese deber ser que, por supuesto, no es" (p.4). 
Con todo, la teoría del delito de Welzel no bastó como herramienta para la solución de nuevos tipos de conflictos que generó la sociedad del riesgo, ya que se constituyeron otro tipo de cuestiones como la contaminación ambiental, la responsabilidad penal de las personas jurídicas por desfalcos millonarios que afectaban a la sociedad en conjunto, y la actualización del papel del derecho penal frente a avances tecnológicos producto de la modernidad tardía o segunda modernidad (Silva Sánchez, 2001). Es así, como se estableció una nueva forma de ver el derecho, la cual cada vez más se aleja de las estructuras lógico-objetivas, estableciendo el paradigma de mayor resonancia en la actualidad: el funcionalismo (Agudelo Betancur, 1998).

Las críticas al funcionalismo no son pocas. Algunos académicos se muestran recelosos del abandono de lo óntico y de la ampliación del derecho a aspectos que no guardan coherencia con la realidad: se ha constituido una segunda vuelta al idealismo. Pabón Gómez (2018) expresa con claridad una de las principales consecuencias de este esquema del delito: “(...) al pretender construir redefiniciones de categorías penales con prescindencia de toda referencia ontológica (Rueda, 2002, p. 409), se apuesta a concebir el delito a partir de la objetiva infidelidad del hombre sistémico al derecho (...)" (p. 308).

A pesar de la aparente disimilitud entre los esquemas y escuelas expuestos, no se puede dejar de lado que todos estos desarrollos teóricos parten de una base normativa y especialmente filosófico-especulativa en común. De donde resulta que no es posible afirmar la existencia de una dicotomía entre estos modelos dogmáticos, sino la continuidad o el menor o mayor grado de apego o desapego a la óntico. La base en común de todos estos planteamientos es el derecho penal liberal (Zaffaroni, 2016).

Los aportes del derecho penal liberal son de importante consideración, tanto así que en la actualidad se puede reconocer claramente en el ejercicio del jurista los principios pensados por Beccaria en el siglo XVIII. Sin embargo, no se puede pasar por alto que la realidad para la que se construyó data de hace dos siglos, por lo que debe ser enfatizado que este fue un derecho desarrollado por la burguesía en ascenso, en un periodo donde la estratificación en Europa era notable. De ahí que la exclusión de cualquier dato social fuese menester para conservar la legitimidad de un sistema legal en una época de bastante inestabilidad política y económica. La lucha contra la imprevisibilidad de la nobleza daba prioridad a sólo uno de los actores (la burguesía), olvidando la gran mayoría de personas que componía la sociedad (Zaffaroni, 2016, p. 54-59).

De esta manera, se comienzan a trazar las características del derecho penal liberal, las cuales persiguen hasta el presente a los ordenamientos jurídicos latinoamericanos. Siendo el deductivismo una de las características principales, ya que, en un estado de conmoción, de cambio, los pensadores iluministas llegaron a la conclusión que la mejor forma de elaboración normativa sería a través de la construcción ideal de un estado racional del que se dedujeran las leyes y sus consecuencias. Era necesaria la legitimación de un sector del poder punitivo para deslegitimar al otro, consagrándose de esta manera la tan anhelada estabilidad burguesa:

(...) el deductivismo y el idealismo del derecho penal liberal, que lleva a los jueces a imponer penas reales en un estado real, pero legitimadas según deducciones de un estado racional imaginario $y$, además, como el imaginario estado racional es inmóvil (como todo lo ideal), lleva a un derecho penal estático en el sentido de una legitimación supuestamente parcial del poder punitivo, pero que parece fijada de una vez para siempre. (Zaffaroni, 2016: 59)

Todo esto permite mirar críticamente las importaciones realizadas en nuestro contexto. Importaciones que no tuvieron en cuenta el carácter político de las diferentes propuestas, ocasionando que en la realidad latinoamericana se introdujera un discurso que permitiera ocultar las falencias y debilidades de las instituciones, como la estratificación y desigualdad de nuestras sociedades.

Con esto, no queremos negar la importancia de algunas de estas elaboraciones dogmáticas en la contención del poder punitivo, ni queremos incurrir en una posición retraída que exalte la capacidad institucional de los países del "primer mundo", lo que se propone este texto es poner de presente que aún en estas realidades, la fundamentación teórica del derecho penal liberal sirve tanto para legitimar discursos que 
distorsionan $\mathrm{u}$ ocultan la realidad como para limitar la barbarie.

No obstante, el primer uso es el que ha tenido mayor preponderancia en la pulsión que existe dentro del derecho, configurándose lo que Zaffaroni describe como "el derecho penal inhumano". Todo lo cual, se trae y se aplica acríticamente en el derecho penal, sirviendo como ejemplo el eufemístico Sistema de Responsabilidad para Adolescentes, que partiendo de categorías naturalizadas construye un entramado jurídico que presume ser dulce, humano y pedagógico.

\section{DESNATURALIZACIÓN DEL CONCEPTO DE INFANCIA}

La década de los 60 resignificó el trabajo de la sociología estadounidense. Hasta la fecha, los trabajos sociológicos simplemente correspondían a esfuerzos institucionales que enfrentaban ciertos problemas desde una perspectiva de política pública positivista: diagnostico, diseño, implementación y evaluación. No obstante, en una década llena de pulsiones y de inquietud filosófica, la Escuela de Chicago sirvió de epicentro para el desarrollo de uno de los trabajos más importantes en el cambio de la criminología etiológica de corte positivista de ese entonces (Becker, 1963).

Howard Becker (1963), interesado en el estudio de la desviación, encontró que las bases del sentido común eran implementadas en las teorías científicas que asumían que los actos que violan normas son inherentemente desviados. Sin embargo, para Becker esta conclusión ignora el carácter político de la desviación, la cual es creada por la sociedad. En otras palabras, los grupos sociales crean la desviación por medio de la expedición de leyes que constituirán en infractor a su quebrantador, lo que conllevará la posterior aplicación de la etiqueta de outsider. Por tanto, la desviación no es una cualidad de la persona infractora, sino una consecuencia de la aplicación de reglas sociales creadas en un determinado contexto social (Becker, 1963: 4-9).

Este descubrimiento científico ha sido extrapolado a otros campos de conocimiento, quedando claro que la asunción de naturalidad de ciertas categorías no es más que un comodín que da una seguridad falsa. De esta manera, se hace necesario revisar todas las clasificaciones, conceptos o discursos que se han dado por sentado y mirarlos desde una perspectiva agnóstica, que reconstruya la realidad desde una genealogía nietzscheana o foucaultiana. Un ejemplo de lo expresado lo da el concepto de "niñez", que muestra la mistificación y naturalización que puede sufrir un concepto, que como éste ha sido asignado con diversas características: fragilidad, necesidad de protección, vulnerabilidad, ternura, entre otros (García Méndez, 2017).

Philippe Aries (1992), en su libro El niño y la vida familiar en el antiguo régimen, siguiendo esta línea de pensamiento, hizo un ejercicio crítico sobre el concepto de "infancia", precisando que antes del siglo XVII la niñez no se había constituido en una categoría, siendo descrita esta etapa de la vida como un periodo de dependencia física previo al desarrollo y entrada a la adultez. Asimismo, Aries relata que el interés por estos humanos en desarrollo, o pequeños hombres como eran distinguidos, constituía un ejercicio moral y no humanista, iniciándose de esta forma una aproximación misericordiosa que vino a ser reforzada con la formación de sentimientos de orden y de vergüenza a mediados del siglo XVI y XVII, lo cual trajo consigo la antes no posible concepción de niños corruptos (García Méndez, 2017: 56-58).

Por otra parte, interesa analizar los diversos discursos que trataron y tratan la problemática de los "niños corruptos", pues siguiendo a Foucault, todo ejercicio de poder requiere de un saber que lo legitime (Gomez Jaramillo \& Silva Garcia, 2015). De ahí que la consolidación de la justicia para jóvenes se acompañe de todo un entramado de racionalizaciones teóricas que se remontan a 1870, fecha en que tuvo lugar en Cincinnati el primer National Congress on Penitentiary and Reformatory Discipline (Rivera, 2005).

Este congreso se propuso superar "viejas" concepciones clásicas del castigo, incorporando con ello todo tipo de elaboraciones criminológicas positivistas, que se centraban en la necesidad de corrección de los infractores, quienes debían contar con espacios adecuados para su rehabilitación, partiendo de la obligación de inclusión de sentencias indeterminadas, ya que la corrección del individuo no se podía estandarizar como lo hacía la escuela liberal clásica, ni podía realizarse en un espacio que no diferenciara hombres, mujeres y adolescentes (Rivera, 2005). 
A partir de esta diferenciación, se edificó el sistema de justicia penal de "menores", que combinó la peligrosidad con la vulnerabilidad, por lo que los "menores" eran etiquetados como necesitados de atención y de control. De alguna manera las medidas de atención se volvieron formas de control, de ahí que se marcara una justificación teórica que se paseaba entre el control punitivo $\mathrm{y}$ el paternalismo tutelar (Garland, 1999). "En síntesis, el «modelo tutelar o de la protección» comportó la más absoluta desprotección de los menores frente al ius puniendi del Estado" (Rivera, 2005, 105).

Citando in extenso, Rivera (2005) caracteriza las primeras leyes estadounidenses de Tribunales Tutelares de menores (basadas en estas teorizaciones) de la siguiente manera:

- Una presunción, iuris et de iuris (sin admisión de prueba en contrario) de inimputabilidad a menores, normalmente, de 16 años

- Los niños y jóvenes fueron considerados como «enfermos a curar», más que como «culpables a corregir»,

- Los Jueces que integraron estos primeros Tribunales Tutelares, debían ser una suerte de Psicólogos o terapeutas, antes que auténticos juristas.

- El presupuesto de la actuación penal no fue sólo el delito cometido sino la «conducta irregular y peligrosa».

- Esas conductas a examinar por los nuevos Tribunales Tutelares no consistieron sólo en los delitos cometidos sino, por ejemplo, en fugas del hogar, alcoholismo, conductas inmorales, vagabundeo, callejeo...

- Se llegó a abogar por la criminalización de conductas no tipificadas como delito,

- En consecuencia, los Tribunales Tutelares fueron ser (sic) competentes sobre niños y jóvenes «viciosos», «fueran o no delincuentes, - La reacción jurídica ya no consistió en una pena - es decir, en un mal- sino en una medida educativa y tutelar - es decir, en un bien-, a fin de que con ella se alcanzara la corrección moral del menor,

- Ahora bien, nada de ello obstaba a la notoria dureza de las medidas (Cuello Calón llegó a justificar el carácter educativo de los «azotes», siempre y cuando, eso sí, se aplicaran con finalidad paternal, como «derecho de corrección»),

- La duración de la medida pasó a ser indefinida, con el único límite de que no sobrepase la mayoría de edad civil. (p.104)

Por otro lado, de la misma manera en que comenzamos a importar el idealismo penal a comienzos del siglo XX, la justicia penal juvenil en los ordenamientos jurídicos latinoamericanos es producto de la actividad importadora de nuestros legisladores, en nuestro medio denominada como sistema de responsabilidad penal para adolescentes. Sin embargo, como la responsabilidad de los adolescentes no se concibió desde el mismo estándar que la responsabilidad penal para adultos, hubo una disociación, permaneciendo por un lado la huella paternalista del positivismo, mientras que por el otro se desarrollaban los principios y garantías brindadas por el derecho penal liberal y la dogmática penal alemana.

En el caso de la antigua justicia de menores, no existía una regulación específica a mediados del siglo XIX en América Latina, por lo que las penas privativas de la libertad eran cumplidas en los mismos centros penitenciarios que los adultos. Esta situación llevó a que los menores ${ }^{8}$ tuvieran que enfrentar una serie de atropellos físicos como sexuales, que llamaron más la atención por su inmoralidad que por una cuestión de humanidad. Frente a esta problemática se decidió, con base en la doctrina de la situación irregular, implementar las primeras leyes para menores en 1919 en Argentina y en 1939 en Venezuela, seguidas paulatinamente por el resto de los países latinoamericanos (García Méndez, 2017: 35-36).

Según García Méndez (2017), la doctrina de la situación irregular se caracteriza por considerar que la infancia es objeto de protección. Además, por la creación de categorías dentro de este concepto. De ahí que se diferenciara a los menores de los niños, niñas y adolescentes, siendo los primeros la población en estado de abandono, desprovista del acceso a la educación o a los servicios de salud. Asimismo, debido al énfasis en los menores vulnerables, el sistema vinculó cuestiones que no respondían a delitos, sino a

8. En este caso se comprende el término "menor" en el contexto de la doctrina de la situación irregular de la que se ha venido hablando (Ariès, 1992, García Méndez, 2017, Escalante, 2009). 
problemas económico-sociales, lo que conllevó a la criminalización de la pobreza y la vulneración de las garantías de la infancia con medidas que respondían a una lógica de todo vale. De modo que el Estado, a través de jueces que parecían más psicólogos paternales que juristas, tenía el deber de suprimir u ocultar las falencias institucionales a partir del castigo dulcificado, que se empezó a denominar como "sanción pedagógica" (Rivera, 2005).

En definitiva, la doctrina de la situación irregular respondió a la incapacidad política de los gobernantes para responder a problemas sociales (Ávila, 2017), quienes con o sin intención utilizaron un discurso de cuño positivista y paternalista -a pesar de que se hubiese abandonado este discurso en el derecho penal de adultos, no paso lo mismo con el de menores- que se centró en el tratamiento y la medicalización de problemas de carácter social, dejando de esta manera desprotegida a la infancia de bajos recursos frente a las medidas "protectoras". En otras palabras, se infringía dolor en nombre del amor y la protección. Esta doctrina propicio la impunidad de los sectores privilegiados de la sociedad, que según la concepción de desamparo no necesitaba de la mano protectora del Estado. En palabras de García Méndez (2017):

Este enfoque específico del desajuste social, producto de un modelo de desarrollo basado en la exclusión, es decir, en la incapacidad política del sistema de universalizar los servicios básicos (salud-educación), tenía en la nueva figura del juez de menores el centro de irradiación de las prácticas concretas. Ungido de una competencia omnímoda penal-tutelar, el juez de menores resulta el encargado de resolver, paternalmente, las deficiencias estructurales del sistema. (p.36)

A pesar de la preponderancia de la doctrina de la situación irregular en el pensamiento jurídico latinoamericano, con la aprobación de nuevos instrumentos internacionales sobre la protección de la infancia y la adolescencia, se fijó que no bastaba que el adolescente acusado hubiese cometido un hecho antisocial, sino que era necesario que la conducta fuese típica, antijurídica y culpable, abandonándose de esta manera la intervención judicial en los casos en que meramente se presentara una situación irregular. En consecuencia, se originó un nuevo paradigma, el paradigma de la protección integral, el cual está integrado por los siguientes instrumentos internacionales: la Convención Internacional sobre los Derechos del Niño; las Reglas Mínimas de las Naciones Unidas para los Jóvenes Privados de la Libertad; las Directrices de las Naciones Unidas para la Administración de la Justicia Juvenil; y las Reglas de Beijing (García Méndez, 2017), todo ello con consonancia y alineado a las formas de constitucionalización del ordenamiento jurídico y de los llamados Neoconstitucionalismos (Guastini, 2009).

A diferencia del paradigma anterior, éste se caracteriza por la noción global de infancia y por el distanciamiento de perspectivas positivistas o paternalistas que trataban el conflicto desde una visión tutelar. Todo esto resultó en un cambio de perspectiva de la infancia como objeto de protección a la infancia como sujeto pleno de derechos, lo cual implicó, por fin, el respeto de los mandatos constitucionales al incorporar las garantías del campo penal en sentido constitucional y de principios garantistas a la responsabilidad penal para adolescentes.

Sin embargo, pese al cambio significativo que trajo consigo el paso de la doctrina de la situación irregular al paradigma de la protección integral. No se puede pasar por alto que la introducción de las garantías del derecho penal significó la inclusión del derecho penal liberal en los asuntos de la responsabilidad penal juvenil. De esto se deriva, que tanto las cualidades como los defectos de este entramado jurídico se ven plasmados en la solución de este tipo de conflictos. Por tanto, el idealismo penal y sus efectos ideológicos en la responsabilidad penal de los adolescentes deben ser mirados de manera cautelosa, con el fin de exponer y atacar la disociación entre el derecho y la realidad, o lo que García Méndez entiende como el surgimiento del paradigma de la ambigüedad, el cual es entendido de la siguiente manera:

El paradigma de la ambigüedad se encuentra muy bien representado por aquellos que, rechazando de plano el paradigma de la situación irregular, no consiguen acompañar las transformaciones reales $\mathrm{y}$ potenciales que se deducen de la aplicación consecuente del paradigma de la protección integral, que considera al niño y al adolescente un sujeto de derechos y no menos, de responsabilidades. (García Méndez, 2017: 275) 
En suma, la adopción del paradigma de la protección integral trajo consigo una nueva visión de la responsabilidad penal para adolescentes, sumado a todo un conjunto de garantías, principios y valores que orientan a este sistema desde los postulados del idealismo penal, lo que ha remarcado la disociación entre la realidad y lo normativo.

Desde el punto de vista de la valoración de la responsabilidad penal propiamente dicha, la implementación del SRPA con base en el código penal de adultos no deja de plantear problemas de aplicación, interpretación y aumento de la complejidad, especialmente en lo que implica la comprensión de las categorías dogmáticas del delito a la luz de los derechos de la infancia y la adolescencia en el marco constitucional. Como se ha planteado de manera permanente en otros escritos (López Betancur \& Escalante, 2019; Escalante, 2020), el SRPA está compuesto por diversas dimensiones, de manera que es un sistema multidimensional, complejo y diferenciado. Cada dimensión comprende un marco axiológico, de principios y normas rectoras. De tal manera, que su lectura debe ser integral $\mathrm{y}$ multidimensional, permanente $\mathrm{y}$ no solo en análisis aislados o de casos especiales o difíciles.

No obstante ello, la dimensión dogmática no es posible desarrollarla en este artículo de investigación, pero si es posible recordar que la aplicación constitucional de los principios y reglas especiales de la infancia y la adolescencia implica una permanente integración normativa que, en el caso de los adolescentes, lleva al análisis que necesariamente debe hacer el intérprete en una integración compleja, no sólo de los principios del proceso penal oral, los principios de la justicia restaurativa o de la infancia y la adolescencia y los principios del derecho penal sustancial. Todo lo cual implica que también debe integrar cada una de las normas rectoras que constituyen el código penal colombiano en armonía con las normas especiales y de manera relevante, con el interés superior del adolescente, en toda su dimensión y correcta comprensión constitucional en el marco del paradigma de la protección integral, con el principio de prevalencia de los derechos de la infancia, la adolescencia y el bloque de constitucionalidad en toda su dimensión convencional. (Escalante, 2020)
De manera que, en el SRPA, el análisis de la responsabilidad y la culpabilidad como principio y como categoría dogmática en la estructura del delito requiere de esa integración normativa constitucional y social, de una valoración de la conducta de los adolescentes en conflicto con la ley penal y del mandato normativo vulnerado, que bien deben tenerse en cuenta, pues su implantación sin un análisis profundo de su aplicación diferencial, también ha llevado a ahondar en el problema de disociación entre el mandato normativo, el constitucional, el de la política penal y el procesal. Para entender mejor la disociación entre ser y deber ser debemos exponer las principales características de este último.

\section{EL DEBER SER DEL SISTEMA DE RESPONSABILIDAD PENAL PARA ADOLESCENTES EN COLOMBIA}

El sistema jurídico colombiano se caracteriza por tener una constitución germinadora de significados sociales útiles para la creación de la realidad social, un instrumento capaz de comunicar, en todos los entornos sociales, los derechos y garantías que hacen viable la interacción entre los ciudadanos y la realización de sus derechos fundamentales. Asimismo, se encuentra permeado e impregnado por las normas constitucionales, cuya característica es la de una constitución "extremadamente invasora" de la producción, interpretación y aplicación de las leyes.

Es evidente su alto grado de constitucionalización, no sólo por la intromisión extrema de la constitución en los ámbitos de producción e interpretación de las leyes sino por la fuerza normativa de la constitución y su aplicación directa. En otras palabras, la Constitución Política es reconocida como máximo criterio de juridicidad, pues marca las pautas de validez del derecho legislado y del derecho aplicado, se trata entonces de una constitución que no solo orienta y condiciona la producción legislativa y las políticas del Estado a través de valores y principios sino también su aplicación judicial en los casos concretos (Guastini, 2003).

No obstante, la Constitución no debe ser entendida como un texto cerrado o como un criterio aislado, sino que debe tener en cuenta los tratados y convenciones internacionales de 
derechos humanos ratificados por el congreso, esto en virtud del bloque de constitucionalidad, establecido en el artículo 93 superior. De esta manera, se protege el derecho de la barbarie legal, ya que se exige otro criterio de validez.

En el caso de la infancia, el artículo 44 superior prescribe que los derechos de los niños priman sobre los derechos de los demás, así que, cuándo se hace referencia a los principios orientadores del sistema, se deben integrar los principios y normas rectoras del código penal, del código de procedimiento penal, de la Constitución, el bloque de constitucionalidad y los principios que cobijan a la infancia y la adolescencia en la Convención de los Derechos de los Niños y las Niñas, lo cual incluye un principio pedagógico y restaurativo. De manera que el SRPA es quizá una de las áreas del derecho más constitucionalizada del ordenamiento jurídico en la actualidad. Este postulado se aplica para todas las categorías normativas, las categorías dogmáticas, las procesales, pedagógicas y de protección integral, de manera que los contenidos y discursos que se tejen alrededor de la adolescencia en conflicto con la ley penal no pueden seguirse haciendo e interpretando de manera aislada, sino conforme a la constitución en sentido material.

Un ejemplo de esto lo da el Sistema de Responsabilidad Penal para Adolescentes (en adelante SRPA), que adopta los mandatos tanto de la Carta política colombiana como de los instrumentos internacionales, al dejar a un lado las enseñanzas de la doctrina de la situación irregular, pues fija como conductas relevantes solamente las que están fijadas en la Ley 599 de 2000. De ahí que se decidiera no perseguir las conductas meramente "inmorales", sino adoptar el análisis escalonado del delito y un concepto de injusto material, esto es, que para que una conducta sea considera jurídico penalmente relevante y merezca una medida sancionatoria, debe ser típica, antijurídica y culpable. Siempre, teniendo en especial consideración la especialidad y el tratamiento diferenciado como principios o mandatos de optimización de una hermenéutica constitucionalmente orientada.

Conviene subrayar que el primer principio va dirigido al establecimiento de institutos, normas, autoridades administrativas especiales (parte orgánica), autoridades de policía y judiciales. Mientras que el segundo principio se refiere a la práctica procesal y a la imposición y ejecución sanción de las conductas jurídicamente relevantes cometidas por los adolescentes, la cual tiene una naturaleza y finalidad completamente diferente a la de los adultos (aspecto sancionatorio).

Tiffer y Llobet (1999) aseguran que la sanción pedagógica debe ser materializada en cada caso en concreto, lo que se consigue con la verificación del restablecimiento y promoción de los derechos, procurando la adecuación de la conducta del adolescente a los valores contenidos en la Constitución Política, tales como el respeto de los derechos fundamentales, la democracia, el autocuidado, la paz, entre otros. Es por esto, que se afirma que la sanción en el SRPA es pedagógica, fin que no sólo se reserva a la sanción sino al proceso mismo (p.87). Citando la literalidad de la Ley 1098 de 2006 (Código de infancia y adolescencia):

Artículo 140. En materia de responsabilidad penal para adolescentes tanto el proceso como las medidas que se tomen son de carácter pedagógico, específico y diferenciado respecto del sistema de adultos, conforme a la protección integral. El proceso deberá garantizar la justicia restaurativa, la verdad y la reparación del daño (...). (Ley 1098, 2006, art.9)

Las sanciones en el SRPA no son homogéneas, pues dependen de la gravedad del delito y de las condiciones y circunstancias que acompañen al adolescente. Es por esto por lo que se han trazado las siguientes: la amonestación, las reglas de conducta, la presentación de servicios sociales a la comunidad, la libertad vigilada, medio semicerrado, y la privación de la libertad, limitando como última medida las dos últimas.

En síntesis, el deber ser del ordenamiento jurídico colombiano en materia de responsabilidad penal para adolescentes, parte de un ordenamiento con un alto grado de constitucionalización, lo que conlleva a la inclusión de instrumentos internacionales en materia de protección de la infancia. Asimismo, se consolida el paradigma de la protección integral que propende por la educación de los adolescentes como por el restablecimiento de sus derechos, todo lo cual responde al respeto de sus garantías y la especialidad y diferenciación de las instituciones como de las sanciones a aplicar, 
reservando las medidas más severas a los casos que más representan daño social, lo cual obedece al principio del interés superior del niño.

\section{EL PROCESO PENAL PARA ADOLESCENTES COMO ESCENARIO COMUNICATIVO Y PEDAGÓGICO}

Una vez visto el origen del derecho penal colombiano, las racionalidades del Sistema de Responsabilidad Penal para Adolescentes y el deber ser de este sistema, son evidentes las falencias estructurales en las que está inmerso. No obstante, este texto no pretende negar los avances del derecho o afirmar su inutilidad. Como se afirmó anteriormente, el derecho penal liberal puede tanto ocultar como brindar herramientas para el respeto y la consolidación de los derechos humanos. Por esta razón, debemos evitar el primer uso, reconociendo que las abstracciones de las fórmulas jurídicas cobijan una lucha entre derecho penal inhumano $y$ el derecho penal humano (Zaffaroni, 2016).

El derecho penal humano encuentra sus cimientos en la Declaración Universal de 1948, la cual estableció que todo ser humano es persona. De ahí que se proponga eliminar cualquier negación o etiqueta de enemigo y superar toda referencia a los adolescentes en conflicto con la ley penal como "peligrosos", "criminales" o "antisociales". A su vez, parte de la necesidad de incorporar datos sociales en la elaboración y realización del debe ser. Todo esto con el propósito de alcanzar las tres coherencias: la coherencia con la realidad, la coherencia normativa y la coherencia política (Zaffaroni, 2016).

Cuando se analiza la responsabilidad penal para adolescentes bajo el tamiz de estas coherencias, es preciso encontrar que el sistema no es coherente con la realidad, la cual determina que la sanción pedagógica termine teniendo los mismos efectos negativos que para el sistema penal de adultos. Ejemplo de esta falta de coherencia lo dan los informes de la Defensoría del pueblo sobre los adolescentes privados de la libertad. (Defensoría del Pueblo, 2015)

En cuanto a la coherencia normativa, lamentablemente, no ha sido clara la postura de todos los actores del poder político y jurídico, pareciera que los derechos de la infancia importaran menos que conservar la "buena" imagen que genera seguir una agenda punitiva, olvidando los mandatos constitucionales e internacionales, lo que lleva a una aplicación disonante con las garantías y principios del sistema. Por último, la coherencia política es nula, el populismo punitivo, los medios de comunicación y los políticos no han hecho más que utilizar como bandera política las afirmaciones del sentido común, ignorando el cimiento esencial del derecho penal humano: todo humano es persona y no instrumentos de opinión pública o proyectos de persona (Escalante, 2018).

Por consiguiente, es dable razonar que la normatividad ha sido insuficiente para alcanzar los fines políticos y jurídicos de este sistema. El énfasis desbordado en la sanción como medida pedagógica ha tenido un papel ideológico, lo que nos induce a pensar que por lograr el deber ser, debemos desconfiar de la sanción privativa de la libertad y encontrar otras formas de alcanzar los propósitos constitucionales sobre la adolescencia.

Sin embargo, el énfasis debe estar mayormente en el proceso y no en la sanción. Desde una perspectiva diferente que entienda al proceso como escenario comunicativo, pedagógico y de garantías, el cual deberá involucrar la interacción de todos los actores con el propósito último de comunicar y servir de escenario pedagógico, reflexivo y orientador para los adolescentes que no hayan interiorizado ciertos valores sociales. Dicho lo anterior, se debe analizar el proceso penal para adolescentes desde tres enfoques: como dispositivo de garantías, como escenario de comunicación y como escenario de acción pedagógica en una interpretación a favor de los adolescentes en conflicto con la ley penal.

El primer enfoque parte de la asunción de que el proceso penal debe ser leído de conformidad con las normas constitucionales y los tratados internacionales sobre derechos humanos, constituyéndose en un escenario de garantías, de comunicación y de reflexión pedagógica permanente por las partes y los diversos actores que en él intervienen, es la manera de realizar el deber ser constitucional anunciado. Por tanto, se incluyen en su marco normativo y analítico, el interés superior del niño y la protección integral, lo cual supone la realización de los derechos a la verdad, justicia y reparación de las víctimas del delito (Corte Constitucional, Sentencia C-209, 2007). 
El segundo enfoque asegura que el proceso penal no es exactamente igual al derecho procesal penal (la ley), ya que el proceso penal es fáctico, es acción, es un conjunto de interacciones y secuencias de actos de comunicación, es un suceso histórico y dinámico, que en cada ejecución es único e irrepetible. (Hassemer \& Muñoz Conde, 1989) De modo que el proceso es comprensión escénica, siendo el derecho procesal penal el encargado de ordenar su curso, las reglas de participación y la limitación de las actuaciones de las partes (garantías), mientras que su desarrollo queda a cargo de los actores, quienes deberán llenar de contenido los enunciados que los rigen. (Hassemer \& Muñoz Conde, 1989, p. 124)

Este escenario de comunicación tiene dos puntos de vista: la socialización de reglas claras y decisiones transparentes para todos los actores e interesados en el proceso; y el deber específico de integrar a los adolescentes infractores a través de la comprensión de todas las etapas como de las finalidades del proceso. Este aspecto, que representa el núcleo de nuestra propuesta, está estrechamente ligado al principio pedagógico del sistema y a la acción educativa que se espera de las partes, de los intervinientes y de los funcionarios judiciales en el proceso penal para adolescentes. Por esta razón, el proceso como escenario de comunicación debe ser configurado y ordenado de forma que todas las personas que intervienen en él, especialmente los adolescentes, puedan conocer exactamente sus derechos de participación e intervención dentro del mismo. Todo lo cual implica también una etapa reflexiva sobre el daño, la víctima y su propio ser como proyecto de vida.

En este contexto se debe integrar también la justicia restaurativa y la necesaria reflexión sobre el daño y la reparación de este como formas de reconocer en el otro a un sujeto de derechos que también merece reconocimiento y respeto en toda su dimensión. Se trata de recuperar a la víctima para el proceso y al victimario para la sociedad, de manera que este escenario así concebido es tanto comunicación, como derecho y reflexión con fines pedagógicos.

Por último, el tercer enfoque se propone darle un mayor peso contra factico al artículo 140 del Código de Infancia y Adolescencia que, al desarrollar la finalidad del sistema, prescribió taxativamente que, en materia de responsabilidad penal para adolescentes, conforme al paradigma de la protección integral, tanto el proceso como las medidas que se tomen son de carácter pedagógico, específico y diferenciado. En este sentido, el principio pedagógico implica no solo la sanción, sino que permea la comprensión escénica del proceso y el rol del adolescente, en el proceso, como sujeto de interacción pedagógica. Esta deducción normativa deriva de los mismos principios $y$ mandatos constitucionales $y$ legales del sistema, sin que sea necesaria una modificación legal, sino su cumplimiento material, más allá de posturas ideológicos y pronunciamientos eufemísticos.

A pesar de ser notoria la irradiación del principio pedagógico en el proceso penal para adolescentes, en el ordenamiento jurídico colombiano los esfuerzos por empujar hacia la realización del deber ser han sido mínimos, ya que todo el empeño por desarrollar los preceptos ha estado dirigido a la ejecución de la sanción y no al proceso. Sirva como ejemplo el "Lineamiento Modelo de Atención para Adolescentes y Jóvenes en Conflicto con la Ley-SRPA" del Instituto Colombiano de Bienestar Familiar (ICBF), el cual orienta todo su esquema únicamente al proceso de atención y a la sanción, olvidando por completo el proceso penal, que incluiría un deber institucional de verificación de la actuación pedagógica de todos los actores del sistema. No obstante, estaría mal desconocer la importancia de este modelo que, aunque no aporta una mirada global del sistema, brinda elementos esenciales para entender su fin pedagógico. Baste, la siguiente cita:

El carácter pedagógico se orienta a la construcción de sujetos críticos, autónomos y sensibles a su entorno. La intencionalidad pedagógica se construye por medio de metodologías que tienen como centro de su acción al propio sujeto, buscando promover en ellos su solidaridad, creatividad, afecto y capacidad para criticar y proponer cambios sustanciales en sus entornos inmediatos como en la sociedad en general (ICBF, 2018: 74).

De esta forma, el ICBF abandona una concepción disciplinaria de la educación para centrar el acto educativo en el adolescente infractor, quien pasará de ser considerado objeto pasivo a sujeto activo del proceso educativo. Aunado a lo anterior, del modelo pedagógico 
planteado en dichos lineamientos es posible deducir un presupuesto que puede ser realizado en las audiencias y no sólo en la ejecución de la sanción pedagógica: la autonomía. Pensemos en el ejercicio de la libertad y en la autonomía moral. Pero ¿qué implica este presupuesto?

La autonomía es lograr el autogobierno de la propia vida, decidir cómo emplear su tiempo, encontrar nuevas formas de significar la vida. También da cuenta de la capacidad del adolescente o joven para interiorizar las normas sociales y los limites en la convivencia inmediata $y$ en espacios comunitarios, no ya como una imposición del otro sino desde la comprensión de su importancia en el ordenamiento social y en la posibilidad de asumir los conflictos como parte de la vida en tanto en la concertación y resolución de los mismos prime el bien común, el acuerdo de cómo queremos vivir en sociedad. (ICBF, 2018: 114)

Así que no es apropiado afirmar que los centros de reclusión para adolescentes cumplen una función pedagogica, ya que la imposición (heterenomia moral) de valores no conlleva a una adopción, sino a la generación de espacios de resistencia (Garland, 1999, p. 204). De ahí que la imposición de disciplinas que nieguen la subjetividad de sus receptores no se refleje en la consecución de los fines del sistema, sino en la adopción de otros valores, conformandose de esta manera subculturas criminales (Baratta, 2002: 70).

La resistencia a los mandatos legales se refuerza por su incomprensión, muchos de los adolescentes que están en el sistema no son informados de la actuación, ni comprenden las implicaciones de su conducta, dejando en el vacío el compromiso por remediar y superar la situación. Por último, otro de los impedimientos que enfrenta el sistema es la comunicación de mensajes no deseados a los adolescentes, quienes se enfrentan ante la majestad presente en el simbolismo de los juicios criminales que, aunque pueda tener resultados positivos en los adultos (Prado, 2004), en los adolescentes sólo causa apatía, incomprensión y distanciamiento del acto educativo.

En definitiva, si el Sistema de Responsabilidad Penal para Adolescentes desea alcanzar los mandatos del deber ser debe poner el enfasis en el proceso como dispositivo de garantías, como escenario de comunicación y como escenario de acción pedagógica, para lo cual deberá ser consecuente materialmente con los mandatos de la constitucion y de los tratados internacionales de derechos humanos. Por otro lado, teniendo en cuenta las enseñanazas de Hassemer, al ser concebido el proceso como comprensión escenica, los espacios donde se llevan a cabo las audiencias, así como la vestimenta de sus actores deberan ser reformados con el proposito de reducir la distancia existente entre los actores y el adolescente en conflicto con la ley. Todo lo cual repercutirá en la reflexion y adopción autonoma de valores por parte del adolescente, que al menos en los casos más graves sabrá por qué es sujeto de la sanción privativa de la libertad, que como hemos venido afirmando en el texto, no cumple los propositos que asegura cumplir.

\section{CONCLUSIONES}

1. La adopción acrítica de las elaboraciones del derecho penal alemán introdujo en la realidad latinoamericana propuestas dogmáticas que conllevaron a la apropiación de los males como de los beneficios del derecho penal liberal, el cual a partir de la construcción ideal de un estado racional deducía (deduce) las leyes y sus consecuencias, negando la inclusión de cualquier dato social. En consecuencia, la realidad latinoamericana introdujo un discurso que permite ocultar las falencias y debilidades de las instituciones, como la estratificación y desigualdad de nuestras sociedades. Todo lo cual, termina en reflejarse en el Sistema de Responsabilidad Penal para Adolescentes, que cobija dos racionalidades diferentes de la cuestión:

1.1. La doctrina de la situación irregular se caracterizó por considerar que la infancia es objeto de protección, vinculando cuestiones que no respondían a delitos, sino a problemas económico-sociales, lo que implicó la criminalización de la pobreza y la vulneración de las garantías de la infancia con medidas que respondían a una lógica de todo vale. En definitiva, esta doctrina respondió a la incapacidad política de los gobernantes para responder a problemas sociales, quienes con o sin intención utilizaron un discurso de cuño positivista y paternalista, que se centró en el tratamiento y la medicalización de problemas de carácter social, dejando de 
esta manera desprotegida a la infancia de bajos recursos frente a las medidas "protectoras", en otras palabras, se infringía dolor en nombre del amor y la protección.

1.2. Ahora bien, el paradigma de la de la protección integral se caracteriza por la noción global de infancia y por el distanciamiento de perspectivas positivistas o paternalistas que trataban el conflicto desde una visión tutelar. Todo esto resultó en un cambio de perspectiva de la infancia como objeto de protección a la infancia como sujeto pleno de derechos, consolidándose el respeto por los mandatos constitucionales al incorporar las garantías del proceso y del derecho penal para adultos a la responsabilidad penal para adolescentes. Sin embargo, la introducción de estas garantías significó la inclusión del derecho penal liberal en los asuntos de la responsabilidad penal para adolescentes. De esto se deriva, que tanto las cualidades como defectos de este entramado jurídico se ven plasmados en la solución de este tipo de conflictos.

2. Por otro lado, el deber ser del ordenamiento jurídico colombiano en materia de responsabilidad penal para adolescentes, parte de un ordenamiento con un alto grado de constitucionalización, lo que conlleva a la inclusión de instrumentos internacionales en materia de protección de la infancia. Además, el paradigma de la protección integral que propende por la educación de los adolescentes como por el restablecimiento de sus derechos, lo cual responde al respeto de sus garantías y a la diferenciación y especialidad de las instituciones como de las sanciones a aplicar, reservando las medidas más severas a los casos más difíciles. En concreto, en el deber ser el Sistema de Responsabilidad Penal para Adolescentes (SRPA) adopta los mandatos tanto de la Carta como de los instrumentos internacionales, al dejar a un lado las enseñanzas de la doctrina de la situación irregular.

3. Sin embargo, las finalidades establecidas en la normatividad que promulgó este sistema no han tenido otro efecto que el de ocultar las falencias estructurales de nuestra sociedad. Los centros de reclusión para adolescentes no llevan a cabo el fin pedagógico de la sanción. Estas condiciones no permiten que el adolescente reflexione sobre sus actos ni que adopte los valores de la democracia, sino que, como en el caso de las cárceles para adultos, inicie o continúe una carrera criminal, sin posibilidades para pensar en un proyecto de vida distinto.

4. En definitiva, el Sistema de Responsabilidad Penal para Adolescentes debe poner el énfasis en el proceso como dispositivo de garantías, como escenario de comunicación y como escenario de acción pedagógica, para lo cual deberá ser consecuente materialmente con los mandatos de la constitución y de los tratados internacionales de derechos humanos. Asimismo, los espacios donde se llevan a cabo las audiencias, así como la vestimenta de sus actores deberán ser reformados con el propósito de reducir la distancia disciplinar existente entre los actores y el adolescente infractor. Esto repercutirá en la reflexión y adopción autónoma de valores por parte del adolescente, que al menos en los casos más graves sabrá por qué es sujeto de la sanción privativa de la libertad, promoviéndose de esta manera un paso de la heteronomía a la autonomía moral.

\section{BIBLIOGRAFÍA}

- Agudelo Betancur, N. (1998). Curso de Derecho Penal Esquemas del Delito. Bogotá : Nuevo foro .

- Ariès, P. (1992). El Niño y la Vida Familiar en el Antiguo Regimen. Madrid: Taurus.

- Ávila, K. (2017). Homicidios en Venezuela: Principales debates y aproximaciones explicativas. Revista Misión Jurídica
10 (12): 117-140. DOI: https://doi. org/10.25058/1794600X.148

- Baratta, A. (2002). Criminología Crítia y Crítica del Derecho Penal. Introducción a la sociología jurídico-penal. Buenos Aires: Siglo veintiuno editores Argentina.

- Becker, H. S. (1963). OUTSIDERS Studies in the Sociology of Deviance. London: Collier 
Macmillan Limited.

- Defensoría del pueblo. (2015). Estado actual de los derechos humanos de las/ los adolescentes privados de la libertad en el Sistema de responsabilidad penal para adolescentes - SRPA. En J. Díaz Soto, M. Hurtado Sáenz, E. Escalante Barreto, E. Gómez Torres, \& L. Rincón León, Estado actual de los derechos humanos de las/ los adolescentes privados de la libertad en el Sistema de responsabilidad penal para adolescentes - SRPA. Bogotá: Defensoría del Pueblo.

- Díaz Mejía, N. (2018). Criminalización del adolescente en el modelo neoliberal: una mirada latinoamericana. En E. Escalante, Política criminal mediática, Populismo penal, Criminología crítica de los Medios y de la Justicia penal (págs. 449-468). Bogotá: Ibáñez-Universidad Nacional de Colombia.

- Donini, M. (2011). El problema del método penal: de Arturo Rocco al Europeísmo judicial*. Revista Nuevo Foro Penal, 49-100.

- Escalante, E. (2009). El Código de la Infancia y la Adolescencia: Elementos para su comprensión e interpretación. En A. Quiroz, \& E. Escalante, Formación integral Ley de la infancia y la adolescencia: análisis $y$ perspectivas (págs. 11-38). Bogotá: Universidad Nacional de Colombia.

- ----.(2018). Justicia penal juvenil: negación de un mandamiento constitucional desde el discurso mediático y político del delito y la defensa social. En E. Escalante, Política criminal mediática, Populismo penal, Criminología crítica de los Medios y de la Justicia penal (págs. 279-430). Bogotá: Ibáñez-Universidad Nacional de Colombia.

- -----(2018). Política Criminal Mediática, Populismo Penal, Criminología Crítica de los Medios y de la Justicia Penal. Bogotá: IbáñezUniversidad Nacional de Colombia.

- -----.(2020). La culpabilidad en el sistema de responsabilidad penal de los adolescentes. Análisis desde la perspectiva dialéctica del derecho penal. En D. Araque Moreno, Estudios Penales en Homenaje a Mario Salazar Marín (págs. 101-142). Bogotá: Ibáñez.

- García, D. y Serrano, O. H. (2008). Evolución del sistema penal acusatorio en el marco del derecho germano, anglosajón y colombiano. Revista Misión Jurídica 1 (1): 97 - 108. DOI: https://doi.org/10.25058/1794600X.4.

- García de Enterría, E. (1994). La Constitución como norma y el Tribunal Constituciona (Tercera ed.). Madrid: Civita.

- García Méndez, E. (2017). Infancia. Buenos Aires: Ediciones Didot.

- Gargarella, R. (2008). De la injusticia penal a la justicia social. Bogotá: Ibáñez - Uniandes.

- Garland, D. (1999). Castigo y Sociedad Moderna Un estudio de teoría. Madrid: siglo veintiuno editores.

- Gómez Jaramillo, A. (2008). Un mundo sin cárceles es posible. México: Ediciones Coyoacán.

- Gomez Jaramillo, A., \& Silva Garcia, G. (2015). El Futuro de la Criminología Crítica. Bogotá: Universidad Catolica de Colombia.

- Grosso García, M. (2019). Reforma y contrareforma del proceso penal sudamericano. El caso colombiano. Bogotá: Ibáñez.

- Guastini, R. (2009). La Constitucionalización del Ordenamiento Jurídico: el Caso Italiano. En M. Carbonell, Neoconstitucionalismo. Madrid: Editorial Trotta.

- Hassemer, W., \& Muñoz Conde, F. (1989). Introducción a la criminología y al derecho penal. Valencia: Tirant lo Blanch.

- ICBF. (2018). Lineamiento Modelo de Atención para Adolescentes y Jóvenes en Conflicto con la Ley-SRPA. Bogotá: Subdirección de Responsabilidad Penal-ICBF.

- Kuhn, T. (2013). La estructura de las revoluciones científicas. México: Fondo de Cultura económica.

- Lombroso, C. (1909). Los Criminales. Barcelona: Centro Editorial Presa. 
- López Betancur, M., \& Escalante, E. (2019). Módulo formación auto-dirigida en el área penal; Sistema de responsabilidad para adolescentes - SRPA. Bogotá: Consejo Superior de la Judicatura - Escuela Judicial Rodrigo Lara Bonilla.

- Martínez A, A. (2018). Infancia y mediatización entre la prescindibilidad, el peligrosismo y el uso mercantil de las infancias y adolescencias. En E. Escalante, Política criminal mediática, Populismo penal, Criminología crítica de los Medios y de la Justicia penal (págs. 469-494). Bogotá: Ibáñez-Universidad Nacional de Colombia.

- Pabón Gómez, G. (2018). De la Doctrina Sistémica Funcionalista del Normativismo Purista. En E. E. Barreto, Problemas Actuales de Derecho Penal General, Libro Homenaje a Luis Carlos Pérez (págs. 297-310). Bogotá: Ibañez.

- Prado, C. (2004). Dos Concepciones del Castigo en torno a Marx. En I. Rivera, Mitologías y Discursos sobre el Castigo. Historia del Presente y Posibles Escenarios (págs. 113 -130). Barcelona: Anthropos.

- Rivera, I. (2005). Política Criminal y Sistema Penal. Viejas y nuevas racionalidades punitivas. Barcelona: Anthropos.

- Rocco, A. (1986). El problema y el método de la ciencia del derecho penal. Bogotá: Temis.

- Romero, A. (2018). Narrativas, punitivismo y control social de adolescentes. En E. Escalante, Política criminal mediática, Populismo penal, Criminología crítica de los Medios y de la Justicia penal (págs. 431448). Bogotá: Ibáñez-Universidad Nacional de Colombia.

- Silva Sánchez, J. M. (2001). La expansión del Derecho penal. Aspectos de la política criminal en las sociedades postindustriales. Madrid: Cuadernos Civitas.

- Tiffer, C., \& Llobet, J. (1999). La Sanción Penal Juvenil y sus Alternativas en Costa Rica. Unicef.

- Vega, P. D. (2011). Apuntes sobre la evolución histórica de la dogmática jurídico-penal. Revista perspectivas de las ciencias económicas y jurídicas, 57-76.

- Welzel, H. (1951). Teoría de la acción finalista. Buenos Aires: Astrea.

- Zaffaroni, E. R. (19 al 21 de agosto de 2015). Memoria Académica. Recuperado el 22 de 5 de 2019, de X Jornadas de Investigación en Filosofía: http://www.memoria.fahce.unlp. edu.ar/trab_eventos/ev.7655/ev.7655.pdf

- Zaffaroni, E. R. (2016). Derecho penal humano y poder en el siglo XXI. Bogotá: Ibañez.

- Zaffaroni, E. R. (2017). Doctrina Penal Nazi, la Dogmática Penal Alemana entre 1933 y 1945. Buenos Aires: Ediar. 